\title{
Simultaneous phase matching of optical parametric oscillation and second-harmonic generation in aperiodically poled lithium niobate
}

\author{
Tolga Kartaloğlu, Z. Gürkan Figen, and Orhan Aytür \\ Department of Electrical and Electronics Engineering, Bilkent University, TR-06533 Bilkent, Ankara, Turkey
}

Received June 5, 2002; revised manuscript received September 10, 2002

\begin{abstract}
We report a simple ad hoc method for designing an aperiodic grating structure to quasi-phase match two arbitrary second-order nonlinear processes simultaneously within the same electric-field-poled crystal. This method also allows the relative strength of the two processes to be adjusted freely, thereby enabling maximization of the overall conversion efficiency. We also report an experiment that is based on an aperiodically poled lithium niobate crystal that was designed by use of our method. In this crystal, parametric oscillation and second-harmonic generation are simultaneously phase matched for upconversion of a femtosecond Ti:sapphire laser to $570 \mathrm{~nm}$. This self-doubling optical parametric oscillator provides an experimental verification of our design method. (c) 2003 Optical Society of America

OCIS codes: $190.2620,190.7220,190.4400,190.4970,190.4410,190.4360$.
\end{abstract}

\section{INTRODUCTION}

In recent years there has been a growing interest in the simultaneous phase matching of two different nonlinear interactions within the same second-order nonlinear crystal. $^{1-29}$ Such simultaneous phase matching allows efficient wavelength conversion of lasers to wavelengths that cannot be reached with a single nonlinear process. For example, the combination of optical parametric oscillation with second-harmonic generation (SHG) in the same second-order nonlinear crystal facilitates efficient conversion to wavelengths shorter than those of the source laser., ${ }^{3,5}$ Even though we can reach the same wavelength range by cascading an optical parametric oscillator (OPO) and a SHG crystal (either external or internal to the OPO cavity), the conversion efficiency of the two-step process is usually low, and the presence of a second crystal increases system complexity. Instead, using a single crystal that is simultaneously phase matched for both parametric oscillation and SHG has proved to be more advantageous. ${ }^{3}$

In second-order nonlinear crystals, the phase-matching condition is most commonly satisfied either by birefringent phase matching (BPM), in which the natural birefringence of the crystal is utilized, ${ }^{30}$ or by quasi-phase matching (QPM), in which periodic domain reversals fabricated into the crystal lead to a grating momentum that cancels the natural phase mismatch. ${ }^{31-33}$ Both $\mathrm{BPM}^{1-4}$ and $\mathrm{QPM}^{8-13}$ have been used for simultaneous phase matching of two separate processes within the same nonlinear crystal. When the BPM method is used, the two phase-matching conditions are simultaneously satisfied for a set of wavelengths and polarization directions, a particular direction of propagation, and a particular temperature. This is the result of a coincidental crossing of the phase-matching curves of the two processes. ${ }^{1-4}$ In the case of QPM, the phase-mismatch term of one process is canceled by the fundamental Fourier component of the grating momentum; the grating period is chosen to this end. The phase-mismatch term of the second process has to coincide with one of the harmonics of the grating momentum for simultaneous phase matching to occur. ${ }^{8-13}$ Both BPM and QPM rely on coincidences to work. Given a pair of source and target wavelengths, there is no guarantee that either method will come up with a suitable crystal design.

When two processes are simultaneously phase matched within the same nonlinear crystal for a given set of wavelengths, the relative strength of the two processes (the ratio of the two effective nonlinear coefficients), is an important parameter that influences the overall conversion efficiency. $^{5,6}$ Neither BPM nor QPM allows any room for the adjustment of this important parameter. If one is lucky enough to achieve simultaneous phase matching for a given set of wavelengths, one just has to live with whatever relative strength these two processes happen to have. This is a restriction that seriously hampers the capabilities of simultaneously phase-matched wavelength conversion systems.

Recently, a number of groups proposed the use of quasiperiodic or aperiodic grating structures to achieve simultaneous phase matching with QPM. ${ }^{14-29}$ One approach has been the utilization of quasi-periodic gratings based on Fibonacci sequences ${ }^{14-22}$; third-harmonic generation (THG $^{15,17,19,21}$ and multiple-peak frequency doubling ${ }^{16}$ (phase-matched SHG for a number of distinct discrete wavelengths) were experimentally demonstrated by use of such gratings. In Fibonacci-based gratings, the locations (in the transform domain) of different Fourier components of the grating momentum are not independent. Therefore, Fibonacci-based gratings also rely on coincidences and cannot be used to cancel any two arbitrary phase-mismatch terms simultaneously. This limitation was alleviated by an extension of Fibonacci-based gratings that allows independent placement of the Fourier 
components. $^{23-25}$ Two-peak frequency doubling ${ }^{24}$ and $\mathrm{THG}^{24,25}$ were experimentally demonstrated by use of these structures. However, neither approach provides a general mechanism to adjust the relative strength of the two simultaneously phase-matched processes. Aperiodic grating structures that are optimized by use of simulated annealing to maximize the conversion efficiency were proposed for multiple-peak frequency doubling, tripling, and parametric amplification. ${ }^{26-28}$ However, this approach does not take pump depletion into account and cannot be expected to yield accurate results. Another method for designing aperiodic grating structures that enables free adjustment of the relative strength of the two processes was proposed ${ }^{29}$ but awaits experimental verification.

Here we describe a simple ad hoc method for designing an aperiodic grating structure to phase match two arbitrary second-order nonlinear processes simultaneously within the same crystal. ${ }^{34}$ This method also allows the relative strength of the two processes to be adjusted freely. We also report an experiment that is based on an aperiodically poled lithium niobate (APLN) crystal that was designed by use of our method. In this crystal, parametric oscillation and SHG are simultaneously phase matched for upconversion of a femtosecond Ti:sapphire laser to $570 \mathrm{~nm}$. This self-doubling OPO provides an experimental verification of our design method.

\section{SIMULTANEOUS PHASE MATCHING}

A second-order $\left[\chi^{(2)}\right]$ nonlinearity results in the coupled interaction of three waves whose frequencies are related by $\omega_{3}=\omega_{1}+\omega_{2} \cdot{ }^{30}$ The wave vector or phase-mismatch term $\Delta k=k_{3}-k_{1}-k_{2}$ (for collinear waves) plays an important role in this interaction; the phase-matching condition $\Delta k=0$ has to be satisfied for efficient conversion of energy from one wave to another. ${ }^{30}$ The particular type of nonlinear interaction that takes place depends on the initial conditions at the input facet of the nonlinear crystal; one could have SHG $\left(\omega_{1}=\omega_{2} \rightarrow \omega_{3}=2 \omega_{1}\right)$, sum-frequency generation $\left(\omega_{1}, \omega_{2} \rightarrow \omega_{3}=\omega_{1}+\omega_{2}\right)$, difference-frequency generation $\quad\left(\omega_{1}, \omega_{3} \rightarrow \omega_{2}=\omega_{3}\right.$ - $\left.\omega_{1}\right)$, or parametric generation $\left(\omega_{3} \rightarrow \omega_{2}, \omega_{1}\right)$.

When two separate processes, say process $a$ and process $b$, are simultaneously phase matched, the phasemismatch terms of both processes,

$$
\begin{aligned}
& \Delta k_{a}=k_{3}-k_{1}-k_{2}, \\
& \Delta k_{b}=k_{6}-k_{4}-k_{5},
\end{aligned}
$$

have to vanish for the same direction of propagation and temperature within the crystal (where $\omega_{6}=\omega_{4}+\omega_{5}$ for process $b$ ). The two processes could have one or two fields in common. Consider the case of THG, for example. ${ }^{10,12,14,15,17-27,29}$ The input wave is at frequency $\omega_{1}=\omega_{2}$; a SHG process generates $\omega_{3}=2 \omega_{1}$. The residual fundamental at $\omega_{4}=\omega_{1}$ is then summed with the second harmonic at $\omega_{5}=\omega_{3}=2 \omega_{1}$ to yield the third harmonic at $\omega_{6}=\omega_{4}+\omega_{5}=3 \omega_{1}$. Another example is the self-doubling OPO. ${ }^{3,5,11,13}$ The input pump wave is at $\omega_{3}$; the OPO generates a signal wave at $\omega_{2}$ and an idler wave at $\omega_{1}$, the specific signal and idler frequencies being determined by the phase-matching condition for the OPO process. The simultaneously phase-matched SHG process generates the second harmonic of the signal, where $\omega_{4}=\omega_{5}=\omega_{2}$ and $\omega_{6}=2 \omega_{2}$. A third example is the cascaded (tandem) OPO, where the signal of an OPO becomes the pump of a second OPO. ${ }^{7}$

In $\mathrm{BPM}$ of a single nonlinear process, the interacting waves have different polarization directions, which is necessary since a monotonic dispersion curve makes it impossible to phase match three waves of the same polarization. When two processes are phase matched simultaneously with BPM, a number of different polarization configurations are possible. In general, these polarization configurations can be grouped into various classes, each class being governed by a different set of coupledmode equations. ${ }^{5,6}$ For common nonlinear crystals, there are only a handful of processes and wavelengths that provide simultaneous phase matching.

In QPM of a single nonlinear process, the interacting waves usually have the same polarization direction. The largest element of the nonlinear coefficient tensor is a diagonal element for common crystals such as lithium niobate, and such a polarization arrangement facilitates the use of the largest nonlinear coefficient $\left(d_{33}\right.$ for lithium niobate). Electric-field poling of the crystal in lithographically selected regions creates domain inversions, resulting in a modulation of the sign of the nonlinear coefficient in the direction of propagation. ${ }^{35}$ The resulting nonlinear coefficient $d(z)=g(z) d_{i j}$ is a function of distance $z$ in the direction of propagation, where $d_{i j}$ is the nonlinear tensor element for the interaction and $g(z)$ is the grating modulation function that can take values only of +1 or -1 . A periodic grating function with period $\Lambda$ can be expressed as a Fourier series:

$$
g(z)=\sum_{p=-\infty}^{\infty} G_{p} \exp (i 2 \pi p z / \Lambda)
$$

where $G_{p}$ represents the Fourier coefficients. QPM is achieved by adjustment of period $\Lambda$ so that the natural phase mismatch $\Delta k_{a}$ is canceled by one of the Fourier components of the grating function, ${ }^{32}$ i.e.,

$$
\Delta k_{a}=\frac{2 \pi}{\Lambda} p
$$

for $p$ th order QPM. In this case the effective nonlinear coefficient that governs the interaction becomes $d_{e}$ $=\left|G_{p}\right|\left|d_{i j}\right|$. The simplest possible grating function is a rectangular wave with a period of $\Lambda$ and a duty cycle of $D_{c}$. To maximize $d_{e}$, it is common practice to set $\Lambda$ to a value that will satisfy Eq. (4) for $p=1$ (first-order QPM) and set $D_{c}=0.5$, in which case $\left|G_{1}\right|=2 / \pi$. Simultaneous phase matching of a second process is possible only if the phase-mismatch term of this second process $\Delta k_{b}$ happens to coincide with one of the nonzero harmonics of the grating modulation function. More generally, higherorder simultaneous phase matching with a periodic grating is possible only when both

$$
\Delta k_{a}=\frac{2 \pi}{\Lambda} p
$$




$$
\Delta k_{b}=\frac{2 \pi}{\Lambda} q
$$

are satisfied for some particular set of $\Lambda$, and integers $p$ and $q$. (Note that if $D_{c}=0.5, p$ and $q$ cannot be even integers, since $G_{p}=0$ for even $p$ in this case.) Even though such circumstances could occur by coincidence, ${ }^{8-13}$ it is not possible to achieve simultaneous phase matching with periodic gratings for arbitrary phase-mismatch terms $\Delta k_{a}$ and $\Delta k_{b}$. Even if one can find a set of $\Lambda, p$, and $q$ to achieve simultaneous phase matching, the interaction will be weak unless either $p=1$ or $q=1$. Another handicap of periodic gratings is that the ratio of the effective nonlinear coefficients

$$
\frac{d_{e b}}{d_{e a}}=\left|\frac{G_{q}}{G_{p}}\right|
$$

cannot be adjusted freely.

An aperiodic grating function can be expressed as

$$
g(z)=\frac{l_{c}}{2 \pi} \int_{-\infty}^{\infty} G(\Delta k) \exp (i \Delta k z) d(\Delta k),
$$

where

$$
G(\Delta k)=\frac{1}{l_{c}} \int_{0}^{l_{c}} g(z) \exp (-i \Delta k z) \mathrm{d} z
$$

is the normalized Fourier transform in the $\Delta k$ domain and $l_{c}$ is the crystal length. Simultaneous phase matching is achieved when $|G(\Delta k)|$ has peaks located at both $\Delta k_{a}$ and $\Delta k_{b}$ in the transform domain. The effective nonlinear coefficients are given by

$$
\begin{aligned}
& d_{e a}=\left|G\left(\Delta k_{a}\right)\right|\left|d_{i j}\right|, \\
& d_{e b}=\left|G\left(\Delta k_{b}\right)\right|\left|d_{i j}\right|,
\end{aligned}
$$

and their ratio is

$$
\frac{d_{e b}}{d_{e a}}=\left|\frac{G\left(\Delta k_{b}\right)}{G\left(\Delta k_{a}\right)}\right| \text {. }
$$

The total energy of the grating function

$$
\int_{-\infty}^{\infty}|G(\Delta k)|^{2} d(\Delta k)=2 \pi / l_{c}
$$

is distributed among all the Fourier components. The fraction of grating energy that is spent toward simultaneous phase matching is

$$
\frac{1}{2 \pi / l_{c}}\left[\int_{\Delta k_{a}-\epsilon / 2}^{\Delta k_{a}+\epsilon / 2}|G(\Delta k)|^{2} d(\Delta k)+\int_{\Delta k_{b}-\epsilon / 2}^{\Delta k_{b}+\epsilon / 2}|G(\Delta k)|^{2} d(\Delta k)\right],
$$

where $\epsilon$ is the width of the Fourier peaks in the $\Delta k$ domain. This quantity should be made as large as possible to maximize the effective nonlinear coefficients. The phase-matching bandwidth $\epsilon$ is equal to the natural phase-matching bandwidth $4 \pi / l_{c}$ regardless of the specific grating function as long as the longest domain length in the crystal is much shorter than $l_{c}$, a condition that is always satisfied. Therefore, maximizing $\left|G\left(\Delta k_{a}\right)\right|^{2}$ $+\left|G\left(\Delta k_{b}\right)\right|^{2}$ achieves the same result as maximizing ex- pression (14). Since the ratio $d_{e b} / d_{e a}$ is fixed ahead of time, maximization of either Fourier component peak assures that the total energy in the wasted components is minimized.

For a given pair of arbitrary phase-mismatch terms $\Delta k_{a}$ and $\Delta k_{b}$, the problem of simultaneous phase matching is to find a grating modulation function $g(z)$ so that

- $g(z)$ can assume values only of +1 and -1 ,

- $|G(\Delta k)|$ has peaks at $\Delta k=\Delta k_{a}$ and $\Delta k=\Delta k_{b}$,

- the ratio $\left|G\left(\Delta k_{b}\right) / G\left(\Delta k_{a}\right)\right|$ is equal to a predetermined constant $\alpha$, and

- $\left|G\left(\Delta k_{a}\right)\right|^{2}$ [or $\left|G\left(\Delta k_{b}\right)\right|^{2}$ ] is as large as possible.

\section{APERIODIC GRATING DESIGN METHOD}

Here we describe a simple ad hoc method for designing an aperiodic grating function $g(z)$ for simultaneous phase matching of two arbitrary second-order nonlinear processes. ${ }^{34}$ Our method is numerical and iterative; we guess at grating function $g(z)$, calculate its Fourier components $G\left(\Delta k_{a}\right)$ and $G\left(\Delta k_{b}\right)$, determine how well the design requirements are satisfied, and modify our initial guess. Various technical restrictions (such as the minimum domain length) are also imposed on $g(z)$ at each iteration.

For a given pair of $\Delta k_{a}$ and $\Delta k_{b}$ values, we start with a continuous-valued function

$$
f(z)=\cos \left(\Delta k_{a} z\right)+A \cos \left(\Delta k_{b} z+\phi\right),
$$

which is composed of the sum of two harmonic functions at $\Delta k_{a}$ and $\Delta k_{b}$ with amplitudes unity and $A$, respectively, and a phase difference $\phi$. At the first iteration, we set the ratio of the two amplitudes to the targeted effective nonlinear coefficient ratio; i.e., $A=\alpha$. We also set phase difference $\phi$ to zero. For this $f(z)$ we calculate $g(z)$ as

$$
g(z)=\operatorname{sgn}[f(z)]
$$

where sgn represents the signum function. In other words, we calculate the roots of $f(z)$ and set these to be the locations of the domain walls along the direction of propagation.

The grating mask used in the fabrication of the poled crystal is specified with a certain spatial resolution. This resolution is $0.05 \mu \mathrm{m}$ in the fabrication process that we use. Therefore, at this stage we round off the domain wall locations to the nearest $0.05-\mu \mathrm{m}$ increment. In addition, domain walls that form after electric-field poling are guaranteed to be uniform only if no two domain walls are closer to each other than a critical distance, typically a few micrometers. We tried to be conservative and took this critical distance to be $5 \mu \mathrm{m}$. In general $g(z)$ can have a number of domains whose lengths are shorter than the critical distance. At this stage we simply discard such domains. In other words, the sign of any domain whose length is less than $5 \mu \mathrm{m}$ is flipped, thereby transforming this and the two adjacent domains into a single domain. We do this at each iteration by sequentially flipping the sign of the shortest domain whose length is less than $5 \mu \mathrm{m}$ until there are no domains left shorter 
than this critical length. This elimination method ensures that the critical length restriction affects the grating minimally.

The resulting $g(z)$ becomes our first guess at a grating function. We then calculate the Fourier transform $G(\Delta k)$. The magnitude of the Fourier transform naturally has peaks located at $\Delta k=\Delta k_{a}$ and $\Delta k=\Delta k_{b}$, in addition to various other components located elsewhere. We calculate the magnitudes of these peaks $\left|G\left(\Delta k_{a}\right)\right|$ and $\left|G\left(\Delta k_{b}\right)\right|$, compare the ratio $\left|G\left(\Delta k_{b}\right) / G\left(\Delta k_{a}\right)\right|$ to $\alpha$, and modify the value of $A$ accordingly for the next iteration in small steps. This iterative method converges to a final $g(z)$ with the desired properties.

This procedure results in an aperiodic grating function $g(z)$ that has relatively high peaks at $\Delta k_{a}$ and $\Delta k_{b}$, no domain lengths smaller than the critical fabrication length, and the desired ratio for the effective nonlinear coefficients. The only question that remains uncertain is whether the resulting effective nonlinear coefficients are as large as possible, i.e., whether $\left|G\left(\Delta k_{a}\right)\right|$ is maximized under the current constraints. We are not aware of any rigorous method that can answer this question. However, we maintain that, as long as the effective nonlinear coefficients are high enough, whether the grating function at hand is optimum is a moot point from a practical point of view. We can get a sense of whether $\left|G\left(\Delta k_{a}\right)\right|$ is high enough by comparing its magnitude with $2 / \pi$, the largest Fourier coefficient of a periodic grating with $D_{c}=0.5$. Since there are two useful components, it makes more sense to compare $\left|G\left(\Delta k_{a}\right)\right|^{2}+\left|G\left(\Delta k_{b}\right)\right|^{2}$ with $(2 / \pi)^{2}$.

We also investigated using the phase difference $\phi$ as another freely adjustable parameter in the iteration process. We found that even though the value of $\phi$ influences both the individual magnitudes and the ratio of the two Fourier components, its effects are usually within a few percent, as long as the crystal length is much larger than the average domain size. For the sake of simplicity, we set $\phi=0$ at the beginning and do not modify this value.

\section{SELF-DOUBLING OPTICAL PARAMETRIC OSCILLATOR}

A self-doubling OPO is based on a nonlinear crystal for which parametric generation and SHG are simultaneously phase matched. A low-loss resonator that is resonant at the signal wavelength forms the self-doubling OPO cavity. Energy is coupled out of the cavity through the simultaneously phase-matched SHG interaction.

\section{A. Coupled-Mode Equations}

For collinear monochromatic plane waves

$$
E_{m}(z, t)=\operatorname{Re}\left\{E_{m} \exp \left[i\left(\omega_{m} t-k_{m} z\right)\right]\right\}, \quad m=1,2,3,
$$

with complex field amplitudes $E_{m}$, the coupled-mode equations that describe a phase-matched second-order interaction are $^{36}$

$$
\frac{\mathrm{d} E_{1}}{\mathrm{~d} z}=-i \frac{\omega_{1} d_{e}}{n_{1} c} E_{3} E_{2}{ }^{*},
$$

$$
\begin{aligned}
& \frac{\mathrm{d} E_{2}}{\mathrm{~d} z}=-i \frac{\omega_{2} d_{e}}{n_{2} c} E_{3} E_{1} *, \\
& \frac{\mathrm{d} E_{3}}{\mathrm{~d} z}=-i \frac{\omega_{3} d_{e}}{n_{3} c} E_{1} E_{2},
\end{aligned}
$$

where $d_{e}$ is the effective nonlinear coefficient and $n_{m}$ represents the refractive indices. A pump field at $\omega_{3}$ and a signal field at $\omega_{2}$ at the input result in an optical parametric amplifier (OPA), where the signal gets amplified and, in the process, an idler field at $\omega_{1}$ is generated. ${ }^{36}$

In an OPA, the lack of an idler field at the input of the crystal results in field solutions whose intensities are independent of the relative phases of the pump and the signal; the generated idler adjusts its phase to compensate for the phase fluctuations in the input pump and signal beams. Utilizing this property, it is possible to convert the three coupled-mode equations for the complex field amplitudes into three real equations. ${ }^{5}$ It is also convenient to define normalized field amplitudes $a_{m}$ such that $a_{m}{ }^{2}$ represents the photon flux densities at each frequency $\omega_{m}$. In doing this, the phases of $E_{m}$ are chosen such that the required phase relation for the OPA is satisfied, and the real and normalized amplitudes $a_{m}$ are defined as

$$
\begin{aligned}
& E_{1}=-i\left(2 \hbar \omega_{1} / n_{1} c \epsilon_{0}\right)^{1 / 2} a_{1}, \\
& E_{2}=\left(2 \hbar \omega_{2} / n_{2} c \epsilon_{0}\right)^{1 / 2} a_{2}, \\
& E_{3}=\left(2 \hbar \omega_{3} / n_{3} c \epsilon_{0}\right)^{1 / 2} a_{3} .
\end{aligned}
$$

The coupled-mode equations for the normalized field amplitudes can then be written in the form

$$
\begin{aligned}
& \frac{\mathrm{d} a_{1}}{\mathrm{~d} z}=\kappa_{a} a_{3} a_{2}, \\
& \frac{\mathrm{d} a_{2}}{\mathrm{~d} z}=\kappa_{a} a_{3} a_{1}, \\
& \frac{\mathrm{d} a_{3}}{\mathrm{~d} z}=-\kappa_{a} a_{1} a_{2},
\end{aligned}
$$

where the coupling constant for the OPA process is defined as

$$
\kappa_{a}=d_{e a}\left(\frac{2 \hbar}{c^{3} \epsilon_{0}}\right)^{1 / 2}\left(\frac{\omega_{1} \omega_{2} \omega_{3}}{n_{1} n_{2} n_{3}}\right)^{1 / 2} .
$$

In the case of SHG, the fundamental field is at $\omega_{4}=\omega_{5}$ and the second-harmonic field at $\omega_{6}=2 \omega_{4}$. Since $a_{4}$ and $a_{5}$ are the same fields, the coupled-mode equations become $^{30}$

$$
\begin{aligned}
& \frac{\mathrm{d} a_{4}}{\mathrm{~d} z}=-\kappa_{b} a_{6} a_{4}, \\
& \frac{\mathrm{d} a_{6}}{\mathrm{~d} z}=\frac{1}{2} \kappa_{b} a_{4}^{2},
\end{aligned}
$$

with the coupling constant

$$
\kappa_{b}=d_{e b}\left(\frac{2 \hbar}{c^{3} \epsilon_{0}}\right)^{1 / 2}\left(\frac{2 \omega_{4}^{3}}{n_{4}{ }^{2} n_{6}}\right)^{1 / 2} .
$$


As in the OPA case, the lack of a second-harmonic field at the input has allowed us to write the coupled-mode equations for real field amplitudes, where $E_{4}$ $=\left(2 \hbar \omega_{4} / n_{4} c \epsilon_{0}\right)^{1 / 2} a_{4}$ and $E_{6}=-i\left(2 \hbar \omega_{6} / n_{6} c \epsilon_{0}\right)^{1 / 2} a_{6}$.

When the two processes are simultaneously phase matched, the signal field is common to the OPA and SHG processes, which become coupled to each other through the signal field. The set of coupled-mode equations that describe this interaction are ${ }^{5}$

$$
\begin{aligned}
& \frac{\mathrm{d} a_{1}}{\mathrm{~d} z}=\kappa_{a} a_{3} a_{2}, \\
& \frac{\mathrm{d} a_{2}}{\mathrm{~d} z}=\kappa_{a} a_{3} a_{1}-\kappa_{b} a_{6} a_{2}, \\
& \frac{\mathrm{d} a_{3}}{\mathrm{~d} z}=-\kappa_{a} a_{1} a_{2}, \\
& \frac{\mathrm{d} a_{6}}{\mathrm{~d} z}=\frac{1}{2} \kappa_{b} a_{2}{ }^{2} .
\end{aligned}
$$

Using these equations it is possible for us to calculate the net single-pass gain experienced by an input signal field in the presence of simultaneous SHG. ${ }^{5}$ When the nonlinear crystal is placed in an optical cavity that is resonant at the signal wavelength, the single-pass signal gain compensates for parasitic linear losses in the cavity at steady state. The resulting intracavity signal field can then be used to calculate the SHG output and the conversion efficiency. $^{5}$ A theoretical analysis of self-doubling OPA systems has revealed that the ratio

$$
\beta=\frac{\kappa_{b}}{\kappa_{a}}=\frac{d_{e b}}{d_{e a}}\left(\frac{2 \omega_{2}{ }^{2} n_{1} n_{3}}{\omega_{1} \omega_{3} n_{2} n_{6}}\right)^{1 / 2},
$$

along with the nonlinear drive

$$
D=\left[\kappa_{a} a_{3}(0) l_{c}\right]^{2},
$$

where $l_{c}$ is the crystal length and $a_{3}(0)$ is the pump field at the input facet of the crystal, uniquely determines the photon conversion efficiency ${ }^{5}$

$$
\eta=2 \frac{a_{6}{ }^{2}\left(l_{c}\right)}{a_{3}{ }^{2}(0)} .
$$

Therefore, in light of Eq. (12) it is important to be able to adjust the relative magnitudes of the Fourier coefficients of the two processes.

\section{B. Crystal Design}

Using our method, we designed gratings for self-doubling OPO that is based on an APLN crystal, with the aim of converting the output of a Ti:sapphire laser at $790 \mathrm{~nm}$ to the visible at $570 \mathrm{~nm}$. Given these pump and secondharmonic wavelengths, the signal and idler wavelengths are 1140 and $2573 \mathrm{~nm}$, respectively. We designed two different gratings with prescribed $\beta$ values of 0.39 and 0.72 . These $\beta$ values were chosen based on our estimate of the nonlinear drive we would achieve in our setup. The 20-mm-long lithium niobate crystal was $x$ cut, and all the beams were polarized along the $z$ axis of the crystal to utilize the highest nonlinear coefficient $\left(d_{33}\right.$
$=-27 \mathrm{pm} / \mathrm{V}$ for $\mathrm{SHG}$ with $1064 \mathrm{~nm}$ as specified in Ref. 37). Lithographic restrictions limited the poled length of the crystal to $18 \mathrm{~mm}$. The aperture size for each grating is $0.47 \mathrm{~mm} \times 0.5 \mathrm{~mm}$, with $0.5 \mathrm{~mm}$ being the crystal thickness. The temperature-dependent Sellmeier equations given in Ref. 38 were used to calculate the refractive indices. The crystal temperature was chosen to be $130{ }^{\circ} \mathrm{C}$ to avoid photorefractive damage. The phasemismatch terms for the OPO and SHG processes at the chosen wavelengths were $\Delta k_{a}=3.06663 \times 10^{5} \mathrm{~m}^{-1}$ and $\Delta k_{b}=7.67070 \times 10^{5} \mathrm{~m}^{-1}$, respectively. The target $\alpha$ values for our two gratings were 0.203 and 0.375 , and our method converged to $A=0.385$ and $A=0.847$ for the $\beta$ $=0.39$ and $\beta=0.72$ gratings, respectively.

For the two resulting gratings, the fractional error in achieving the prescribed $\beta$ values was within $2 \times 10^{-5}$, much smaller than what is necessary to influence the resulting conversion efficiency appreciably. When we calculated $\beta$ using Eq. (35), we took the dispersion in the nonlinear coefficients into account by employing Miller's rule. ${ }^{37}$

There are 1756 domains in each grating. The smallest domain length is $7.7 \mu \mathrm{m}(6.1 \mu \mathrm{m})$, whereas the largest domain length is $15.2 \mu \mathrm{m}(14.8 \mu \mathrm{m})$ for the first (second) grating. The average domain length for both gratings is $10.3 \mu \mathrm{m}$. Figure 1 shows the length of the inverted and noninverted domains for the two gratings. Figure 2 is an expanded diagram that illustrates domains with domain numbers in the 178-202 range for the first grating ( $\beta$ $=0.39$ ). The aperiodic nature of the grating function is evident.

Figure 3 shows the magnitude of the normalized Fourier transform $[|G(\Delta k)|]$ of the grating modulation functions for the two grating structures. For the first grating ( $\beta=0.39$ ), the fractional errors in placing the first and second Fourier transform peaks at $\Delta k_{a}$ and $\Delta k_{b}$ $\left[\mid(\text { peak location })_{a, b}-\Delta k_{a, b} \mid / \Delta k_{a, b}\right]$ are $2.9 \times 10^{-6}$ and $3.1 \times 10^{-6}$, respectively; whereas for the second grating $(\beta=0.72)$, the corresponding fractional errors are $6.1 \times 10^{-6}$ and $2.1 \times 10^{-6}$. For both gratings, these errors correspond to a shift of the order of $10^{-3} \mathrm{~nm}$ in the

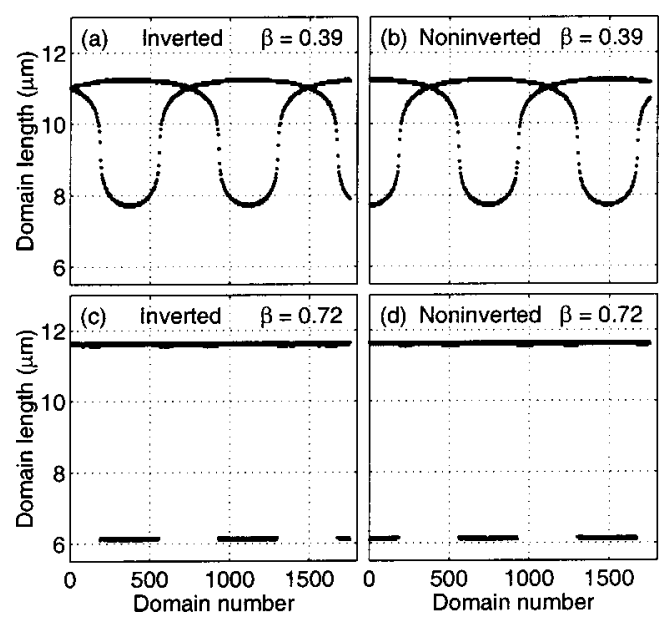

Fig. 1. Lengths of (a) inverted and (b) noninverted domains of the first grating $(\beta=0.39)$ and $(c)$ inverted and (d) noninverted domains of the second grating $(\beta=0.72)$ as functions of domain number. 


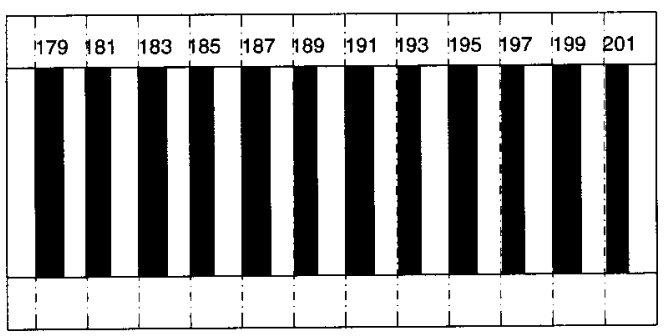

Fig. 2. Expanded diagram illustrating a segment of the first grating $(\beta=0.39)$. Black (white) stripes represent inverted (noninverted) domains. The grid lines are equally spaced.
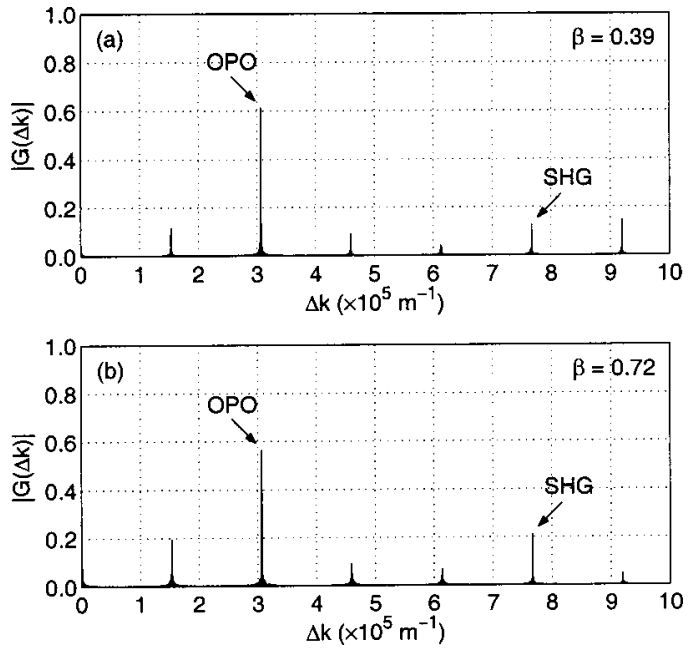

Fig. 3. Magnitude of the normalized Fourier transform $|G(\Delta k)|$ for the (a) first ( $\beta=0.39)$ and (b) second ( $\beta=0.72)$ gratings.

prescribed wavelengths of the pump, signal, and secondharmonic beams.

When the newer temperature-dependent Sellmeier equations given in Ref. 39 are used, the phase-matched pump, signal, and second-harmonic wavelengths with these gratings are off by $0.48,0.34$, and $0.17 \mathrm{~nm}$, respectively.

The total useful Fourier components $\left|G\left(\Delta k_{a}\right)\right|^{2}$ $+\left|G\left(\Delta k_{b}\right)\right|^{2}$ are $0.39(\beta=0.39)$ and $0.36(\beta=0.72)$. Comparing these values with $(2 / \pi)^{2}=0.41$, we note that the gratings designed using our method yield effective nonlinear coefficients that are almost as high as possible.

\section{Experimental Results}

Our APLN crystal was designed with an intracavitypumped cw self-doubling OPO in mind, hence the relatively long crystal length. However, by constructing a simple externally pumped femtosecond self-doubling $\mathrm{OPO}$, we wished to demonstrate the validity of our design method.

The experimental setup shown in Fig. 4 is based on a singly resonant ring cavity formed by four mirrors that are highly reflective at the signal wavelength. Mirrors M1 and M2 are 100-mm radius-of-curvature concave, and M3 and M4 are flat. The APLN crystal is placed in an oven that is kept at a temperature of $130{ }^{\circ} \mathrm{C}$ and positioned at the intracavity focus between M1 and M2. The crystal has antireflection coatings for the pump, signal, and second-harmonic wavelengths on both surfaces. A mode-locked Ti:sapphire laser with 180-fs-long pulses at a repetition rate of $76 \mathrm{MHz}$ provides the pump beam at 790 $\mathrm{nm}$. The pump beam is focused with a lens (L) of $50-\mathrm{mm}$ focal length and enters the cavity through M1. The focused pump has a $62-\mu \mathrm{m}$ diameter (measured) and the lowest-order transverse mode of the cavity has a $60-\mu \mathrm{m}$ diameter (calculated) at the crystal. We achieved synchronization of the signal and pump pulses by moving M3 with a piezoelectrically controlled mount. Mirror M2 was designed to transmit the second-harmonic beam and to reflect the pump beam. The residual (depleted) pump beam is separated from the second-harmonic beam at M2, and leaves the cavity through M3. A dichroic beam splitter (DBS) separates the second-harmonic beam from the idler beam at the output.

When the pump beam is tuned to a wavelength other than the design wavelength of $790 \mathrm{~nm}$, the signal beam that resonates inside the cavity assumes a value that keeps the OPO process phase matched (if possible). In this case the SHG process is not phase matched, and only a weak parasitic second-harmonic beam is produced. Since there is no output coupler at the signal wavelength, the OPO cavity is severely undercoupled. When the pump beam is tuned to $790 \mathrm{~nm}$, the SHG process becomes simultaneously phase matched with the OPO process, and a strong second-harmonic beam is produced. This reduces the intracavity signal power, since the SHG process draws power from the signal beam. We measured the wavelengths of the pump, signal, and secondharmonic beams to be consistent with those initially chosen. Figure 5 shows the signal and second-harmonic spectra for the second grating ( $\beta=0.72$ ) when the pump wavelength is $790 \mathrm{~nm}$. The spectra for the first grating $(\beta=0.39)$ are similar.

Figure 6 shows the power conversion efficiency as a function of input pump power for both gratings. The threshold of the self-doubling OPO with the $\beta=0.72$ grating is $17 \mathrm{~mW}$. This device produces $32 \mathrm{~mW}$ of output

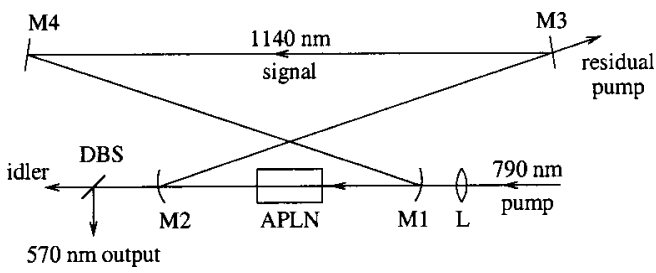

Fig. 4. Femtosecond self-doubling OPO setup.

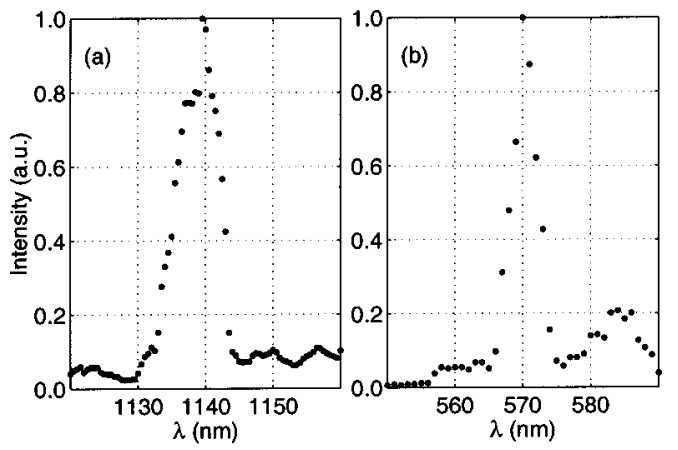

Fig. 5. (a) Signal and (b) second-harmonic spectra at a pump wavelength of $790 \mathrm{~nm}$ for the second grating $(\beta=0.72)$. 


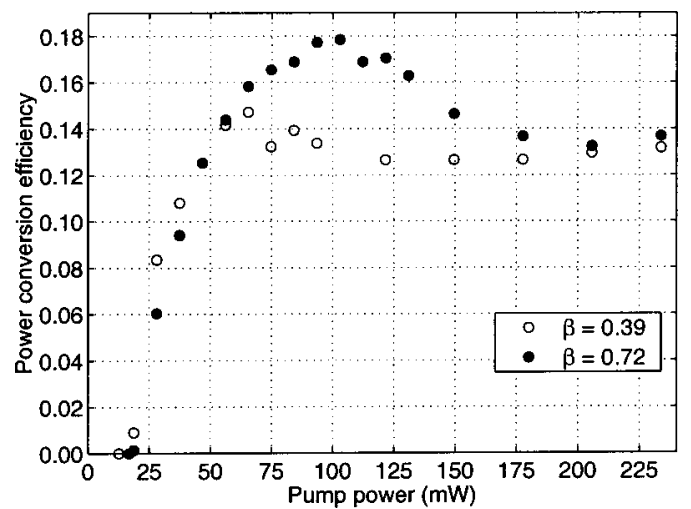

Fig. 6. Power conversion efficiency as a function of the pump power for the first $(\beta=0.39$, open circles) and second ( $\beta$ $=0.72$, filled circles) gratings.

power at the second-harmonic wavelength when the pump power is $234 \mathrm{~mW}$, corresponding to a power conversion efficiency of $13.7 \%$. The maximum power conversion efficiency is $17.9 \%$ at $103 \mathrm{~mW}$ of pump power. This corresponds to a photon conversion efficiency [see Eq. (37)] of $25.7 \%$. For the self-doubling OPO with the $\beta=0.39$ grating, the maximum second-harmonic output power is $31 \mathrm{~mW}$ at a pump power of $234 \mathrm{~mW}$, corresponding to a $13.2 \%$ power conversion efficiency. The maximum power conversion efficiency is $14.7 \%$ at $65 \mathrm{~mW}$ of pump power, corresponding to a photon conversion efficiency of $21.2 \%$. The general shape of the efficiency curves in Fig. 6 is consistent with the plane-wave results of class-A selfdoubling OPOs as reported in Ref. 5 .

Group velocities of the pump, signal, idler and secondharmonic pulses propagating inside the APLN crystal are all different. This group-velocity mismatch (GVM) results in a spatial separation of these pulses as they propagate. This effect has profound consequences on the behavior of the femtosecond self-doubling OPO. The effective interaction length is limited to distances dictated by GVM. Calculated ${ }^{38}$ inverse GVM terms for the pump-signal, pump-idler, signal-idler, and secondharmonic-signal pulses are 222, 286, 64, and $699 \mathrm{fs} / \mathrm{mm}$, respectively. The effective interaction length of the pump and signal pulses in the OPO process is approximately $0.8 \mathrm{~mm}$. Even for such a short interaction length, we achieve large pump depletion $(>50 \%)$, indicating a strong pump-signal interaction. However, the relatively high GVM between the signal and the second-harmonic pulses is the limiting factor for the overall conversion efficiency. Another consequence of GVM is a severe broadening of the second-harmonic pulses. A signal pulse generates second-harmonic power as it propagates over the whole length of the crystal; the second harmonic generated at earlier portions travel slower than the signal pulse and fall behind as a new second harmonic is generated further down the crystal. We measured the secondharmonic output pulse width to be $2.4 \mathrm{ps}$. This value is of the same order of magnitude with the total delay between the signal and the second-harmonic pulses over the entire length of the crystal.

Photon conversion efficiencies that reach $26 \%$ are a strong indication that the OPO and SHG interactions are simultaneously phase matched in the APLN crystal. De- tuning the pump wavelength by less than $0.5 \mathrm{~nm}$ from the peak reduces the second-harmonic output by more than $90 \%$.

\section{CONCLUSION}

In conclusion, the method described in this paper facilitates the design of aperiodic grating structures to quasiphase match two arbitrary second-order nonlinear processes (simultaneously) within the same crystal. This method is much simpler than previous approaches to the same problem. Since the relative strength of the two processes can be adjusted freely, the conversion efficiency of the overall process can be optimized. The selfdoubling OPO experiment reported in this paper provides experimental verification of our method.

\section{ACKNOWLEDGMENTS}

This research was supported in part by the Turkish Scientific and Technical Research Council (Tubitak) under grant 197-E050 and in part by the Bilkent University Research Fund EE-01-03.

O. Aytür's e-mail address is aytur@ee.bilkent.edu.tr.

\section{REFERENCES}

1. R. A. Andrews, H. Rabin, and C. L. Tang, "Coupled parametric downconversion and upconversion with simultaneous phase matching," Phys. Rev. Lett. 25, 605-608 (1970).

2. V. Petrov and F. Noack, "Frequency upconversion of tunable femtosecond pulses by parametric amplification and sumfrequency generation in a single nonlinear crystal," Opt. Lett. 20, 2171-2173 (1995).

3. T. Kartaloğlu, K. G. Köprülü, and O. Aytür, "Phase-matched self-doubling optical parametric oscillator," Opt. Lett. 22, 280-282 (1997).

4. K. G. Köprülü, T. Kartaloğlu, Y. Dikmelik, and O. Aytür, "Single-crystal sum-frequency-generating optical parametric oscillator," J. Opt. Soc. Am. B 16, 1546-1552 (1999).

5. O. Aytür and Y. Dikmelik, "Plane-wave theory of selfdoubling optical parametric oscillators," IEEE J. Quantum Electron. 34, 447-458 (1998).

6. Y. Dikmelik, G. Akgün, and O. Aytür, "Plane-wave dynamics of optical parametric oscillation with simultaneous sumfrequency generation," IEEE J. Quantum Electron. 35, 897-912 (1999).

7. G. T. Moore, K. Koch, M. E. Dearborn, and M. Vaidyanathan, "A simultaneously phase-matched tandem optical parametric oscillator," IEEE J. Quantum Electron. 34, 803810 (1998).

8. S. D. Butterworth, P. G. R. Smith, and D. C. Hanna, "Picosecond Ti:sapphire-pumped optical parametric oscillator based on periodically poled $\mathrm{LiNbO}_{3}$," Opt. Lett. 22, 618620 (1997).

9. K. C. Burr, C. L. Tang, M. A. Arbore, and M. M. Fejer, "High-repetition-rate femtosecond optical parametric oscillator based on periodically poled lithium niobate," Appl. Phys. Lett. 70, 3341-3343 (1997).

10. O. Pfister, J. S. Wells, L. Hollberg, L. Zink, D. A. Van Baak, M. D. Levenson, and W. R. Bosenberg, "Continuous-wave frequency tripling and quadrupling by simultaneous threewave mixings in periodically poled crystals: application to a two-step 1.19-10.71- $\mu$ m frequency bridge," Opt. Lett. 22, 1211-1213 (1997).

11. C. McGowan, D. T. Reid, Z. E. Penman, M. Ebrahimzadeh, W. Sibbett, and D. H. Jundt, "Femtosecond optical paramet- 
ric oscillator based on periodically poled lithium niobate," $\mathrm{J}$ Opt. Soc. Am. B 15, 694-701 (1998).

12. G. Z. Luo, S. N. Zhu, J. L. He, Y. Y. Zhu, H. T. Wang, Z. W. Liu, C. Zhang, and N. B. Ming, "Simultaneously efficient blue and red light generations in a periodically poled $\mathrm{LiTaO}_{3}$, Appl. Phys. Lett. 78, 3006-3008 (2001).

13. X. P. Zhang, J. Hebling, J. Kuhl, W. W. Rühle, and H. Giessen, "Efficient intracavity generation of visible pulses in a femtosecond near-infrared optical parametric oscillator," Opt. Lett. 26, 2005-2007 (2001).

14. J. Feng, Y. Y. Zhu, and N. B. Ming, "Harmonic generations in an optical Fibonacci superlattice," Phys. Rev. B 41, 5578$5582(1990)$

15. S. N. Zhu, Y. Y. Zhu, and N. B. Ming, "Quasi-phase-matched third-harmonic generation in a quasi-periodic optical superlattice," Science 278, 843-846 (1997).

16. S. N. Zhu, Y. Y. Zhu, Y. Q. Qin, H. F. Wang, C. Z. Ge, and N. B. Ming, "Experimental realization of second harmonic generation in a Fibonacci optical superlattice of $\mathrm{LiTaO}_{3}$," Phys. Rev. Lett. 78, 2752-2755 (1997).

17. Y. Y. Zhu, R. F. Xiao, J. S. Fu, G. K. L. Wong, and N. B. Ming, "Third harmonic generation through coupled secondorder nonlinear optical parametric processes in quasiperiodically domain-inverted $\mathrm{Sr}_{0.6} \mathrm{Ba}_{0.4} \mathrm{Nb}_{2} \mathrm{O}_{6}$ optical superlattices," Appl. Phys. Lett. 73, 432-434 (1998).

18. X. Liu, Z. Wang, J. Wu, and N. Ming, "Characterization of third-harmonic generation in Fibonacci optical superlattices," Phys. Rev. A 58, 4956-4960 (1998).

19. Y. Q. Qin, Y. Y. Zhu, S. N. Zhu, G. P. Luo, J. Ma, and N. B. Ming, "Nonlinear optical characterization of a generalized Fibonacci optical superlattice," Appl. Phys. Lett. 75, 448450 (1999).

20. Y. B. Chen, Y. Y. Zhu, Y. Q. Qin, C. Zhang, S. N. Zhu, and N. B. Ming, "Second-harmonic and third-harmonic generation in a three-component Fibonacci optical superlattice," J. Phys. Condens. Matter 12, 529-537 (2000).

21. Y. B. Chen, C. Zhang, Y. Y. Zhu, S. N. Zhu, H. T. Wang, and N. B. Ming, "Optical harmonic generation in a quasi-phasematched three-component Fibonacci superlattice $\mathrm{LiTaO}_{3}$," Appl. Phys. Lett. 78, 577-579 (2001).

22. Y. Y. Zhu and N. B. Ming, "Dielectric superlattices for nonlinear optical effects," Opt. Quantum Electron. 31, 10931128 (1999)

23. K. F. Kashi and A. Arie, "Multiple-wavelength quasi-phasematched nonlinear interactions," IEEE J. Quantum Electron. 35, 1649-1656 (1999).

24. K. F. Kashi, A. Arie, P. Urenski, and G. Rosenman, "Multiple nonlinear optical interactions with arbitrary wave vector differences," Phys. Rev. Lett. 88, 023903 (2002).

25. C. Zhang, H. Wei, Y. Y. Zhu, H. T. Wang, S. N. Zhu, and N. B. Ming, "Third-harmonic generation in a general two- component quasi-periodic optical superlattice," Opt. Lett. 26, 899-901 (2001).

26. B. Y. Gu, B. Z. Dong, Y. Zhang, and G. Z. Yang, "Enhanced harmonic generation in aperiodic optical superlattices," Appl. Phys. Lett. 75, 2175-2177 (1999).

27. B. Y. Gu, Y. Zhang, and B. Z. Dong, "Investigations of harmonic generations in aperiodic optical superlattices," J. Appl. Phys. 87, 7629-7637 (2000).

28. Y. Zhang and B. Y. Gu, "Optimal design of aperiodically poled lithium niobate crystals for multiple wavelengths parametric amplification," Opt. Commun. 192, 417-425 (2001).

29. H. Liu, Y. Y. Zhu, S. N. Zhu, C. Zhang, and N. B. Ming, "Aperiodic optical superlattices engineered for optical frequency conversion," Appl. Phys. Lett. 79, 728-730 (2001).

30. R. W. Boyd, Nonlinear Optics (Academic, San Diego, Calif., 1992).

31. J. A. Armstrong, N. Bloembergen, J. Ducuing, and P. S. Pershan, "Interactions between light waves in a nonlinear dielectric," Phys. Rev. 127, 1918-1939 (1962).

32. M. M. Fejer, G. A. Magel, D. H. Jundt, and R. L. Byer, "Quasi-phase-matched second harmonic generation: tuning and tolerances," IEEE J. Quantum Electron. 28, 26312654 (1992).

33. R. L. Byer, "Quasi-phasematched nonlinear interactions and devices," J. Nonlinear Opt. Phys. Mater. 6, 549-592 (1997).

34. T. Kartaloğlu, Z. G. Figen, and O. Aytür, "A self-doubling optical parametric oscillator based on aperiodically-poled lithium niobate," in 2001 IEEE/LEOS Annual Meeting Conference Proceedings (Institute of Electrical and Electronics Engineers, Piscataway, N.J., 2001), pp. 243-244.

35. L. E. Myers, R. C. Eckardt, M. M. Fejer, R. L. Byer, W. R. Bosenberg, and J. W. Pierce, "Quasi-phase-matched optical parametric oscillators in bulk periodically poled $\mathrm{LiNbO}_{3}$," J. Opt. Soc. Am. B 12, 2102-2116 (1995).

36. R. A. Baumgartner and R. L. Byer, "Optical parametric amplification,” IEEE J. Quantum Electron. QE-15, 432-444 (1979).

37. D. A. Roberts, "Simplified characterization of uniaxial and biaxial nonlinear optical crystals: a plea for standardization of nomenclature and conventions," IEEE J. Quantum Electron. 28, 2057-2074 (1992).

38. G. J. Edwards and M. Lawrence, "A temperaturedependent dispersion equation for congruently grown lithium niobate," Opt. Quantum Electron. 16, 373-375 (1984).

39. D. H. Jundt, "Temperature-dependent Sellmeier equation for the index of refraction, $n_{e}$, in congruent lithium niobate," Opt. Lett. 22, 1553-1555 (1997). 